\section{Wirt-orientierte Therapien bei Influenza - Was heißt das?}

\author{
S. Ludwig ${ }^{1}$ \\ ${ }^{1}$ Institut für Molekulare Virologie (IMV), Zentrum für Molekular- \\ biologie der Entzündung (ZMBE), Westfälische-Wilhelms Uni- \\ versität Münster
}

Die Influenza gehört immer noch zu den großen Seuchen der Menschheit und verursacht nicht nur epidemische Seuchenzüge bei Mensch und Tier, sondern kann auch Pandemien auslösen, die im letzten Jahrhundert Millionen von Menschenleben gekostet haben. Alle Pandemien wurden von Virustypen verursacht, die ganz oder in Teilen von Erregern aus der Tierwelt, aus Vögeln und Schweinen stammten. Influenza ist eine klassische Zoonose; das natürliche Reservoir von Influenza-Viren liegt in wildlebenden Wasservögeln mit einer riesigen genetischen Vielfalt. Daher können InfluenzaViren auch nicht ausgerottet werden. Es ist vielmehr nötig, effiziente Interventionsstrategien zu finden.

Die beste Möglichkeit, sich vor einer Grippe zu schützen, ist nach wie vor die Impfung. Dies gilt natürlich nur dann, wenn dem Impfstoff eine gute Vorhersage zugrunde liegt, er also eine ausreichende Immunität gegen die aktuell zirkulierenden Viren induziert. Deutlich wurde dies unter anderem beim Auftreten der pandemischen Grippe von 2009, ausgelöst durch neue Varianten von Influenza-Viren des Subtyps H1N1, gegen die die saisonalen Impfstoffe unwirksam waren. Damals wurde ein generelles Problem besonders evident: Für die Frühphase einer Pandemie ist die Impfung keine Option, da es mindestens 4-6 Monate dauert, bis genügend Impfstoff gegen ein neu aufgetauchtes Virus zur Verfügung steht. Daher sind sich Experten einig, dass ein dringender Bedarf an antiviralen Medikamenten besteht, um für eine Pandemie gewappnet zu sein.

Unser derzeitiges Arsenal an Influenza-Medikamenten ist leider sehr begrenzt und beschränkt sich auf zwei Wirkstoffgruppen, M2-Ionenkanalblocker (z. B. Amantadin und Rimantadin) und Neuraminidase-Inhibitoren (z.B. Tamiflu und Relenza). Der Vorteil dieser Medikamente, der direkte Angriff auf Bestandteile des Viruspartikel, ist gleichzeitig auch ein Nachteil, denn die Viren können gegen einen direkten Angriff sehr schnell resistente Varianten ausbilden. Aufgrund der Erfahrung mit diesen zugelassenen Substanzen muss man davon ausgehen, dass alle Medikamente, die einen Influenza-Virus direkt angreifen, früher oder später durch Resistenzbildung unwirksam werden. Hier bedarf es also einer neuen Strategie. Die Suche nach neuen antiviralen Ansätzen gegen Influenza ist daher ein sehr aktives Forschungsfeld (Übersicht in: [6]). Jedes Virus braucht Zellen eines Organismus, um sich zu replizieren. Dies bedeutet, dass Viren die Zellen für ihre eigenen Zwecke manipulieren und missbrauchen, um eine effektive Vermehrung zu gewährleisten. Dies ist jedoch gleichzeitig eine Achillesferse und bietet die Möglichkeit, den Viren in den Zellen ihre Vermehrungsgrundlage zu rauben, indem man die für die Vermehrung essenziellen zellulären Faktoren inhibiert. Diese Strategie hat den großen Vorteil, dass ent- sprechende Inhibitoren breit wirksam sein sollten, und dass Resistenzbildung weitgehend verhindert wird, da das Virus die fehlende zelluläre Aktivität nicht ersetzen kann. Gerade in den letzten Jahren hat dieser Ansatz zunehmend Interesse gefunden, was insbesondere an der Entwicklung von neuartigen Screening-Methoden liegt, die helfen, zelluläre Faktoren einzeln zu manipulieren und so ihre Relevanz für die Virusvermehrung zu evaluieren [7].

Von der Vielzahl an zellulären Proteinen, die unter anderem aus diesen Screenings hervorgegangen sind, sind zwei funktionelle Gruppen besonders hervorzuheben. Zur erfolgreichen Infektion muss das Virus mehrere zelluläre Barrieren wie die Plasmamembran und die Kernmembran durchdringen. Daher sind zelluläre Faktoren, die an diesen Prozessen beteiligt sind, besonders interessante Zielpunkte, da hier das Virus direkt mit der Zelle interagieren muss. Eine weitere wichtige Gruppe an Proteinen sind solche, die an der virusinduzierten intrazellulären Signaltransduktion beteiligt sind. Im ruhenden Lungenepithelgewebe sind diese Signalwege hauptsächlich in infizierten Zellen aktiv. Inhibitoren würden daher präferenziell in infizierten Zellen und nicht in den umliegenden ruhenden Epithelzellen wirken, was die Gefahr von Nebenwirkungen einschränkt.

Arbeiten der letzten Jahre haben gezeigt, dass es in der Tat sowohl in Zellkultur als auch im Tiermodell möglich ist, zelluläre Proteine und Enzyme, die essenziell für die Influenza-Virusreplikation sind, zu hemmen und somit die Virusvermehrung effizient und ohne großes Nebenwirkungspotenzial zu blockieren. Dies soll hier am Beispiel von zwei virusinduzierten Signalwegen, der mitogenen Raf/MEK/ERK-Kinase-Kaskade und dem IKK/NF-kappaB-Modul illustriert werden. Die Raf/MEK/ERK-Kaskade wird durch Akkumulation von neu gebildeten viralen Hemagglutinin-Glykoproteinen in der Plasmamembran in PKC-abhängiger Weise aktiviert und kontrolliert den aktiven Export des neu synthetisierten viralen Genoms, welches in Form von Ribonukleoproteinkomplexen (RNPs) vorliegt $[5,8,12]$.

Der IKK/NF-kappaB-Signalweg reguliert die virusinduzierte Expression proapoptotischer Faktoren wie Fas, TRAIL oder FasL, welche in auto- und parakriner Weise zu einer Aktivierung von apoptotischen Caspasen führen. Diese Caspasen spalten in einer frühen Phase der viralen Apoptoseinduktion Proteine der Kernporen. Dabei werden zwar Proteine, die am aktiven Kernexport beteiligt sind, zerstört, die veränderte Kernpore erlaubt aber dann die freie Diffusion von größeren Proteinkomplexen durch die Kernmembran. Das Virus nutzt diesen passiven Mechanismus als Alternative, um die große Menge an neu-gebildeten RNPs bei abnehmender Funktion des aktiven Kernexports zu späten Zeiten des Replikationszyklus aus dem Zellkern zu bringen $[13,14]$.

Beide Signalwege regulieren also auf verschiedene Weise sequenziell den Kernexport von RNPs. Hemmung der Signalwege trifft damit das Virus an der Achillesverse eines Membranüberschreitenden Schritts und führt entsprechend zu einer Retention der RNP im Zellkern und somit zur Blockierung der Virusvermehrung $[2,9,12]$.

Für Hemmstoffe beider Signalwege konnte weiterhin gezeigt werden, dass sie keine evidenten toxischen Wirkungen in Zellkultur oder im Mausmodell aufweisen [1,4], gegen alle 
bislang getesteten Influenza-Viren wirksam sind und und nicht zur Bildung resistenter Virusvarianten führen [2,5]. Darüber hinaus blockieren die Substanzen auch die überschießende Zytokinantwort, den sogenannten Zytokinsturm, der als immunpathologischer Mechanismus oft zu Problemen bei schweren Influenza-Krankheitsverläufen führt $[2,10]$. Einige Hemmstoffe der besagten Signalkaskaden befinden sich bereits in klinischer Entwicklung für andere Krankheiten wie Entzündungen oder Tumorerkrankungen und haben dort überraschend gute Verträglichkeit in klinischen Studien am Menschen gezeigt (Übersicht in [11]). In jüngsten Studien wurde demonstriert, dass diese klinisch geprüften oder z.T. bereits zugelassenen Inhibitoren in Zellkultur und im Tiermodell gegen die verschiedensten Influenza-Viren antiviral aktiv sind und auch synergistisch mit dem Virus-gerichteten Medikament Tamiflu wirken [3]. Aufgrund der vielversprechenden Daten konnte auch bereits eine erste klinische Studie der Phase II mit einem NF-kappaB inhibierenden Wirkstoff gegen schwere Influenza initiiert werden [www.clinicaltrialsregister.eu/ctr-search/trial/2012-004072-19/DE].

Interessenkonflikte: In Bezug auf den Inhalt der Präsentation bestehen keine Interessenkonflikte.

\section{Literatur}

1 Droebner K, Pleschka S, Ludwig S et al. Antiviral activity of the MEK-inhibitor U0126 against pandemic H1N1 v and highly pathogenic avian influenza virus in vitro and in vivo. Antiviral Res 2011; 92: 195-203

2 Ehrhardt C, Ruckle A, Hrincius ER et al. The NF-kappaB inhibitor SC75741 efficiently blocks influenza virus propagation and confers a high barrier for development of viral resistance. Cell Microbiol 2013; 15: $1198-1211$

3 Haasbach E, Hartmayer C, Planz O. Combination of MEK inhibitors and oseltamivir leads to synergistic antiviral effects after influenza $A$ virus infection in vitro. Antiviral Res 2013; 98: 319-324

4 Haasbach E, Reiling SJ, Ehrhardt $C$ et al. The NF-kappaB inhibitor SC75741 protects mice against highly pathogenic avian influenza A virus. Antiviral Res 2013; 99: 336-344

5 Ludwig S, Wolff T, Ehrhardt C et al. MEK inhibition impairs influenza B virus propagation without emergence of resistant variants. FEBS Lett 2004; 561: 37-43
6 Ludwig S, Zell R, Schwemmle M et al. Influenza, a One Health paradigm novel therapeutic strategies to fight a zoonotic pathogen with pandemic potential (2014). Available online, DOI: 10.1016/j.jmm. 2014.08.016

7 Ludwig S. Will omics help to cure the flu? Trends Microbiol 2014; 22: $232-233$

8 Marjuki H, Alam MI, Ehrhardt C et al. Membrane accumulation of influenza $A$ virus hemagglutinin triggers nuclear export of the viral genome via protein kinase Calpha-mediated activation of ERK signaling. J Biol Chem 2006; 281: 16707-16715

9 Mazur I, Wurzer WJ, Ehrhardt C et al. Acetylsalicylic acid (ASA) blocks influenza virus propagation via its NF-kappaB-inhibiting activity. Cell Microbiol 2007; 9: 1683-1694

10 Pinto R, Herold S, Cakarova L et al. Inhibition of influenza virus-induced NF-kappaB and Raf/MEK/ERK activation can reduce both virus titers and cytokine expression simultaneously in vitro and in vivo. Antiviral Res 2011; 92: 45-56

11 Planz 0 . Development of cellular signaling pathway inhibitors as new antivirals against influenza. Antiviral Res 2013; 98: 457-468

12 Pleschka S, Wolff T, Ehrhardt C. Influenza virus propagation is impaired by inhibition of the Raf/MEK/ERK signalling cascade. Nat Cell Biol 2001; 3: $301-305$

13 Wurzer WJ, Ehrhardt C, Pleschka S et al. NF-kappaB-dependent induction of tumor necrosis factor-related apoptosis-inducing ligand (TRAIL) and Fas/FasL is crucial for efficient influenza virus propagation. J Biol Chem 2004; 279: 30931 - 30937

14 Wurzer WJ, Planz O, Ehrhardt C et al. Caspase 3 activation is essential for efficient influenza virus propagation. EMBO J 2003; 22: 2717-2728

Bibliografie

Dol http://dx.doi.org/10.1055/s-0033-1358037

Drug Res 2014; 64, Suppl. 1: S22-S23

(c) Georg Thieme Verlag KG Stuttgart · New York .

ISSN 2194-9379

Korrespondenzadresse

Stephan Ludwig

Westfälische Wilhelms-Universität Münster

von-Esmarch-Straße 56

48149 Münster

ludwigs@uni-muenster.de 